\title{
Exemplifying definitions: a case of a square
}

\author{
Rina Zazkis • Roza Leikin
}

Published online: 30 July 2008

(C) Springer Science + Business Media B.V. 2008

\begin{abstract}
In this study we utilize the notion of learner-generated examples, suggesting that examples generated by students mirror their understanding of particular mathematical concepts. In particular, we explore examples generated by a group of prospective secondary school teachers for a definition of a square. Our framework for analysis includes the categories of accessibility and correctness, richness, and generality. Results shed light on participants' understanding of what a mathematical definition should entail and, moreover, contrast their pedagogical preferences with mathematical considerations.
\end{abstract}

Keywords Examples $\cdot$ Learner-generated examples $\cdot$ Example spaces $\cdot$ Definitions Necessary and Sufficient conditions $\cdot$ Prospective teachers $\cdot$ Secondary mathematics teachers

\section{Background: learner generated examples and example spaces}

The centrality of examples in teaching and learning mathematics has been long acknowledged. This special issue is an additional testimony to researchers' interest in the importance of examples and the significant role they play in mathematics. Within a variety of educational uses of examples in mathematics, we view examples as "illustrations of concepts and principles" (Watson and Mason 2005, p. 3). The specific concept of interest is that of a mathematical definition and it is considered through its specific instantiation: a definition of a square. Our study is inspired by Watson and Mason's (2005) notions of learner-generated examples and example spaces.

"Learner-generated examples" is a teaching strategy of asking learners to construct their own examples of mathematical objects under given constraints. Watson and Mason claim,

R. Zazkis $(\bowtie)$

Faculty of Education, Simon Fraser University, Burnaby, British Columbia, V5A 1S6, Canada

e-mail: zazkis@sfu.ca

R. Leikin

Faculty of Education, University of Haifa, Haifa, 31905, Israel

e-mail: rozal@construct.haifa.ac.il 
and provide ample evidence, that examples generated by learners serve as a powerful pedagogical tool for enhancing the learning of mathematics at a variety of levels.

In our prior research (Zazkis and Leikin 2007) we suggested that this pedagogical tool can be used as a research tool. That is to say, by examining examples generated by participants, researchers may draw inferences about their knowledge, both subject matter knowledge and pedagogical content knowledge. We developed an explicit framework, described below, for evaluating examples generated by participants.

Watson and Mason (2005, p.76) introduced the idea of example spaces, which are collections of examples that fulfill a specific function. They distinguished between several kinds of example spaces:

- Situated (local), personal (individual) example spaces, triggered by a task, cues and environment as well as by recent experience;

- Personal potential example space, from which a local space is drawn, consisting of a person's past experience (even though not explicitly remembered or recalled), and which may not be structured in ways which afford easy access;

- Conventional example space, as generally understood by mathematicians and as displayed in textbooks, into which the teacher hopes to induct his or her students;

- A collective and situated example space, local to a classroom or other group at a particular time, that acts as a local conventional space.

For our purpose the notion of conventional example space needs further refinement. In our prior research (Zazkis and Leikin 2007) we found significant differences between what is "generally understood by mathematicians" and what is "displayed in textbooks". For example, $\sqrt{17}$ or $\sqrt{117}$ definitely belong to the example space of irrational numbers, as understood by mathematicians, but these examples are almost never used in textbooks or instructional interactions, where $\sqrt{2}$ serves as a generic example. As such, we wish to refine Watson and Mason's category of 'conventional example spaces', distinguishing between 'expert' example spaces, that display rich variety of expert knowledge and 'instructional' example spaces, as displayed in textbooks and most often used in instruction. Further, we note that expert example spaces include unconventional examples in addition to the conventional ones.

The distinctions between different example spaces have been acknowledged in our development of a framework, that allows for making inferences about participants' knowledge by analyzing their example spaces, either personal or collective, and comparing them, at times implicitly, to the conventional example spaces. In our study we employ this framework for analyzing the examples participants provided for a definition of a square as a lens on their mathematical and pedagogical knowledge.

In what follows we first focus on definitions in teaching and learning mathematics and illustrate an expert example space for a definition of a square. Next we describe the particulars of our study. We then share with the reader how our personal awareness was increased and how our sensitivity was sharpened. This "transformation in the being of the researchers" is, according to Mason (1998, p. 357), one of the most significant products of mathematics education research.

\section{On definitions in teaching and learning mathematics}

The importance of mathematical definitions in teaching and learning mathematics is irreducible. The definition of a concept, once determined in a curriculum, influences the 
approach to teaching mathematics, the learning sequence, the set of theorems and proofs. Consequently, definitions, and the ways in which they are presented to students, shape the relationship between a concept image and a concept definition, forming an essential part of one's knowledge structure that affects the learner's thinking processes (Tall and Vinner 1981; Vinner 1991).

We consider definitions of mathematical concepts, the underlying structures of the definitions and the process of defining to be fundamental components of the subject matter knowledge of teachers of mathematics. This study stems from the standpoint that teachers' personal knowledge of mathematical definitions affects (a) their curricular decisions regarding the way mathematical concepts are taught, and (b) their pedagogical conception of the ways in which students may or may not learn these concepts. This standpoint follows multiple studies on the role of definitions in secondary school mathematics (Borasi 1992; De Villiers 1998; Pimm 1993; Tall and Vinner 1981; Zaslavsky and Shir 2005) and in professional development programs for mathematics teachers (Cooney and Wilson 1993; Leikin and Winicky-Landman 2000, 2001; Tirosh and Even 1997; van Dormolen and Zaslavsky 2003; Winicky-Landman and Leikin 2000).

Leikin and Winicki-Landman (2000, 2001; Winicky-Landman and Leikin 2000) analyzed the role of equivalent mathematical statements that can serve as definitions of a mathematical concept. They distinguished between mathematical and didactical characteristics of mathematical definitions. Based on the works of well-known mathematicians (e.g., Khinchin 1968, Poincare 1909/1952, Solow 1984, and Vinner 1991) they pointed out logical principles that should be fulfilled in defining mathematical concepts: (a) defining is giving a name: the statement used as a definition presents the name of the concept and this term (name) appears only once in this statement; (b) a definition establishes necessary and sufficient conditions for the concept; (c) in defining the new concept, only previously defined concepts may be used; (d) the set of necessary and sufficient conditions must be minimal; and (e) a definition is arbitrary. We acknowledge that the principle of minimality is debatable and we consider this issue in detail in Section 4.1.1.

Square is a fine example of a mathematical concept having a rich set of statements that may serve as its definition. Figure 1 presents a partial set of definitions of a square, drawn from the expert example space.

Statements 1-3 consider a square as a specific type of a quadrilateral with respect to the properties of its sides and angles. In definitions 4-9 the reference set for a square is another specific quadrilateral (i.e., a rectangle, a rhombus, a parallelogram). Statements 10 and 11 define a square as a symmetrical quadrilateral while definitions 12 and 13 consider a square as a locus of points in a plane.

By examining the logical connections between the statements related to a concept, an equivalence class of defining statements may be established. Every statement that belongs to this class may be chosen arbitrarily as a definition, when other statements of this class are theorems that constitute necessary and sufficient conditions for the concept. However, Poincare (1909/1952) pointed out that in teaching mathematics, satisfying these logical principles is a necessary but not sufficient condition for using a statement as a definition. It is important for the learning process that a chosen definition should be didactically suitable. Each didactic decision is based on the relationship between the nature of mathematics and the nature of the learning process. A didactically suitable definition of a new concept should rely only on the concepts known to the learner. To support learners' intellectual development the definition should belong to the ZPD of a learner (Vygotsky 1982). It is also desirable that the definition should be intuitive (Fischbein 1987; Mariotti and Fischbein 1997) and elegant (Vinner 1991; Van Dormolen and Zaslavsky 2003). 


\section{A square is}

1) a regular quadrilateral

2) a quadrilateral with all the angles and all the sides equal

3) a quadrilateral with all the sides equal and an angle of $90^{\circ}$

4) a rectangle with equal sides

5) a rectangle with perpendicular diagonals

6) a rhombus with equal angles

7) a rhombus with equal diagonals

8) a parallelogram with equal adjacent angles and equal adjacent sides

9) a parallelogram with equal and perpendicular diagonals

10) a quadrilateral having 4 symmetry axes

11) a quadrilateral symmetric under rotation by $90^{\circ}$

12) the locus of all the points in a plane for which the sum of the distances from two given perpendicular lines is constant

13) the locus of all the points in a plane for which the maximum of the distances from two given perpendicular lines is constant

Fig. 1 Definitions of a square from an expert example space

Research in mathematics education has repeatedly demonstrated that both high school students and undergraduate students experience difficulties in interpreting new definitions, and in using definitions appropriately, and that they do not fully appreciate the role of definitions in solving problems or creating proofs (e.g., Dahlberg and Housman 1997, Edwards and Ward 2004, and Zaslavsky and Shir 2005).

We believe that definitions generated by prospective secondary mathematics teachers may serve as a lens for examining their understanding of what a mathematical definition entails as well as their understanding of the specific concepts involved. Thus, examples generation serves in this study as a novel research tool and its implementation is presented below.

\section{The study}

\subsection{The tasks}

Task 1 presented to the participants of the study was:

Give as many examples as possible for a definition of a square.

Participants were invited to respond in writing with no time constraints for completing the task. We have chosen the concept of a square for several reasons: First, it is one of the most familiar concepts in the school mathematics curriculum. Second, the concept is well suited to the task as there is a variety of well known mathematical statements that may serve as a definition of a square.

Task 2, administered a week after task 1, included a list of 24 examples for a definition of a square, some drawn from the expert example space and most chosen from the 
responses of the participants. Participants were invited first to judge individually the validity of each example and then compare their decision in small groups. Whole class discussion addressed the items of disagreement. The goal of Task 2 was to elicit participants' ideas that guided their responses to Task 1 as well as to enrich their example spaces.

\subsection{The setting}

The tasks were presented to a group of 40 prospective secondary mathematics teachers during their final course towards teaching certification. The academic background of the participants included either a Bachelor's degree in Mathematics (major) or a Bachelor's Degree in Science or Engineering with a significant coursework in mathematics (equivalent of a 'minor').

In a prior lesson the notion of equivalent definitions was addressed. It was mentioned that mathematical concepts rather often have several equivalent definitions, and the choice of a definition useful for a particular task is determined by the context. As an example, a perpendicular bisector of a line segment was defined as a line perpendicular to the segment and passing through the midpoint of this segment, and as a locus of points equidistant from two points that are endpoints of the segment.

\subsection{Framework for analysis}

As mentioned above, our framework is a tool for analyzing personal or collective example spaces based on (a) accessibility and correctness, (b) richness, and (c) generality (Zazkis and Leikin 2007). In what follows we introduce these criteria and explain how they were implemented in analyzing Task 1.

\subsubsection{Accessibility and correctness}

In this category we consider whether examples satisfy the conditions of the task and whether they are generated with ease or with a struggle. Specifically, in our analysis of the correctness of teacher-generated examples for a definition of a square we relate to the logical structure of the mathematical statement and minimality. Correct logical structure of a definition includes a set of conditions for the concept that is necessary and sufficient. Exemplifying concept definition by a set of conditions that is necessary but not sufficient or sufficient but not necessary is a manifestation of logical difficulties. Such difficulties may relate to the lack of understanding of the specific concept and its critical features or lack of understanding of the concept of definition itself. For example, defining a square as "a geometrical object with four equal sides and four angles of $90^{\circ}$ " includes necessary but not sufficient conditions and, as such, may indicate misunderstanding of the role of definition in mathematics: A square is a particular case of the set of objects defined by this statement (see Fig. 2).

In contrast, some examples may include specific properties that are unnecessary conditions for the object and are present only in specific cases. For example, the object defined by "a quadrilateral that has four angles of $90^{\circ}$ and four sides of $2 \mathrm{~cm}$ each" is definitely a square, but the statement cannot be used as a definition as it includes sufficient but not necessary conditions. Inability to distinguish between defining and non-critical features of the concept demonstrates the lack of understanding of the general notion of a definition. 
Fig. 2 Example of a polygon with four equal sides and four angles of $90^{\circ}$

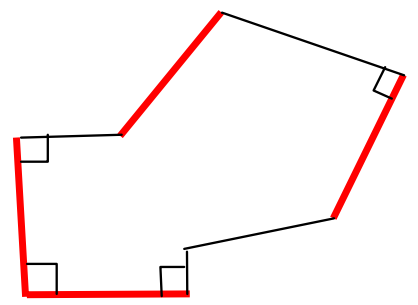

\subsubsection{Richness}

In this category we consider whether the examples vary in type and structure, and whether they are situated in a particular context or drawn from a variety of contexts. Specifically, in our analysis of the participant-generated examples, definitions that extend the conventional-instructional example space are considered as rich. In the case of a definition of a square we identify statements that attend to properties other than sides and angles as examples of a rich example space. For example, definitions 12 and 13 in Fig. 1 are viewed as rich according this criterion.

\subsubsection{Generality}

In this category we consider whether the examples are general or specific. We use caution, noting the overwhelming preference in mathematics education towards general explanations or solutions over the specific ones. We noted (Zazkis and Leikin 2007) that while "some general examples, characterizing classes of objects, may be seen as an indication of mathematical understanding, other general examples may point to deficiencies in understanding. In other words, the 'generality' may serve as a generator, but may also serve as a protective shield" (p. 21). However, in the particular case of definitions, the degree of desirable generality is embedded in the task itself: the definition should relate to a general object of a square, rather than a specific square.

In this study we consider prospective teachers' generated examples for a definition of a square and address two interrelated questions:

1. To what extent do examples generated by participants reveal their understanding of the mathematical concept of a square and the meta-mathematical concept of a definition?

2. To what extent is the framework designed for analyzing examples helpful to this end and how can it be refined or extended to serve this purpose?

\section{Findings: Task 1}

Overall, in responses to Task 1, participants generated 155 statements as examples for a definition of a square. The number of examples provided by an individual varied between two and eight, and the majority of participants listed three or four statements. As the task was presented following previous classroom activities in the context of geometry, it was assumed by the instructor that the concept of a square would be interpreted as related to a 
geometric shape. However, only 140 out of 155 examples of definitions generated by the participants explicitly referred to geometry.

Out of the remaining 15, four definitions were related to the concept of power and concerned "a number multiplied by itself", whereas the other 11 related to a square in a real life context. These included the following examples:

A square is

- A type of a dance

- A boring person

- A carpenter's tool used to measure right angles

- A central meeting place with a fountain.

Since no participant provided the real life context statements exclusively, we believe that those examples were presented only after the participants exhausted their ideas for possible examples of definitions of the geometric shape.

Our analysis focuses on the 140 geometry-related statements generated by the participants as examples for a definition of a square. Rather than sharing with the reader all the definitions, in what follows we classify them according to the different criteria of our framework and provide illustrative examples in each category.

\subsection{Accessibility and correctness}

In the 'accessibility' category we intended to consider the relative ease or difficulty with which examples are generated and the number of examples that participants provide. However, the method of data collection in this study (unlimited time for written response) did not allow for the analysis of the former. Further, consideration of the number of the provided examples is complicated by the fact that both correct and incorrect examples were generated. That is why the discussion in this section is focused on 'correctness'.

When examining the correctness of the examples provided by the teachers we distinguished between appropriate and inappropriate statements. We classified as appropriate 85 out of 140 statements that included necessary and sufficient conditions for a square and thus their use as definitions was legitimate. Accordingly, we classified as inappropriate 55 examples of definitions that included necessary but not sufficient conditions, sufficient but not necessary conditions, or conditions that were neither necessary nor sufficient. A summary of these categories is provided in Table 1.

\subsubsection{Appropriate rigorous examples of definitions}

Among the appropriate examples we distinguished between rigorous and non-rigorous definitions, where rigorous definitions are minimal, or barely-not-minimal, and include accurate mathematical terminology. (The reason for taking into account "barely-not-minimal" examples, as distinguished from significantly-not-minimal, is illustrated and explained below).

Out of 85 appropriate examples, 71 conformed to these standards of mathematical rigor. Statements categorized as examples of rigorous definitions included the following:

A square is

A-1: A rectangle with all sides equal

A-2: A rhombus with a right angle

A-3: A parallelogram with a right angle and all sides of equal length 
Table 1 Correctness of the participants' examples $(n=140)$ for the definition of a square

\begin{tabular}{|c|c|c|}
\hline $\begin{array}{l}\text { Example of a } \\
\text { definition }\end{array}$ & Conditions & Type of example \\
\hline $\begin{array}{l}\text { Appropriate } \\
(\mathrm{n}=85)\end{array}$ & $\begin{array}{l}\text { Necessary and sufficient } \\
(\mathrm{n}=41)\end{array}$ & $\begin{array}{l}\text { Rigorous minimal }(\mathrm{n}=41) \\
\text { Rigorous barely-not-minimal }(\mathrm{n}=30) \\
\text { Non-rigorous: considerably not-minimal, and/or } \\
\text { improper terminology }(\mathrm{n}=14)\end{array}$ \\
\hline \multirow[t]{2}{*}{$\begin{array}{l}\text { Inappropriate } \\
(\mathrm{n}=55)\end{array}$} & $\begin{array}{l}\text { Necessary but not } \\
\text { sufficient }(n=43)\end{array}$ & $\begin{array}{l}\text { Listing properties of a square }(n=10) \\
\text { More general specific quadrilateral }(n=16) \\
\text { Not necessarily a quadrilateral: improper original } \\
\text { object }(n=17)\end{array}$ \\
\hline & $\begin{array}{l}\text { Sufficient but not } \\
\text { necessary }(n=5) \\
\text { Not necessary and not } \\
\text { sufficient }(n=7)\end{array}$ & $\begin{array}{l}\text { Restriction of location, orientation } \\
\text { or size } \\
\text { Inappropriate shape or drawing }\end{array}$ \\
\hline
\end{tabular}

A-4: A rectangle whose diagonal bisects angles

A-5: A rhombus with four $90^{\circ}$ angles

A-6: A rhombus and a rectangle

A-7: A regular quadrilateral

We note that some of the above statements actually lack minimality: In A-1 and A-3 it is sufficient to mention two adjacent equal sides, rather than 'all sides'; in A-5 only one angle of $90^{\circ}$, rather than 'all angles', is sufficient to define a square. We considered these definitions as barely-not-minimal. The decision to consider these statements as examples of rigorous definitions was based on the use of these statements by multiple educational resources such as textbooks and mathematical dictionaries. For example, the Wolfram MathWorld dictionary (Weinstein 2007b) — known as "the web's most extensive mathematics resource"-_defines a square as

"a geometric figure consisting of a convex quadrilateral with sides of equal length that are positioned at right angles to each other".

Note in this definition the reference to right angles (plural), which defies the minimality condition. Further, there is no need for specifying a 'convex' quadrilateral since there is no concave quadrilateral with equal sides. Additionally we need to admit that in many cases barely-not-minimal definitions are more elegant than minimal ones: Consider for example A-7 or A-6 versus "a rectangle with two adjacent sides equal". We further acknowledge that there is no consensus on the minimality requirement for a definition among mathematicians as well as among mathematics educators (Linchevsky, Vinner and Karsenty 1992; Vinner, Linchevski and Karsenty 1993; Zaslavsky and Shir 2005).

Of interest to mention here, that Vasco (2006) classified the conditions in the "families" of definitions of polygons, considering (a) the number of sides, (b) congruence of sides and (c) either congruence of angles or specific measures of angles. In his scheme Definition 2 in Fig. 1 is labeled as ' $4-4-4$ ' definition, that is, it requires a polygon with four sides, four equal sides and four equal angles. In a similar fashion, Definition 3 in Fig. 1 is coded as '44-1' definition, requiring four sides, four equal sides and at least one $90^{\circ}$ angle. Vasco suggested that ' $4-3-2$ '- polygon with four sides, at least three of them equal forming at least two right angles - is yet another minimal definition for a square. 
Overall, 41 of 71 rigorous definitions were identified as minimal, whereas 30 of 71 as barely-not-minimal. What follows are additional examples of barely-not-minimal definitions of a square generated by the participants:

A square is

A-8: A rectangle whose diagonals bisect angles (one diagonal is sufficient)

A-9: A parallelogram with a $90^{\circ}$ angle and all the sides equal (two adjacent sides equal is sufficient)

A-10: A four-sided polygon having all sides equal and all angles equal $90^{\circ}$ (one angle of $90^{\circ}$ is sufficient)

\subsubsection{Appropriate but not rigorous examples of definitions}

The remaining 14 statements that we classified as appropriate but not rigorous were either significantly-not-minimal and/or utilized inaccurate mathematically terminology. A serious redundancy and convolution of definition and properties is exemplified in the following statements:

A square is

A-11: A shape that has four sides of equal length with angles between adjoining sides equal to $90^{\circ}$, giving a total interior angle measure of $360^{\circ}$

A-12: A closed figure with four equal sides that have the two equal length diagonals crossing each other at a right angle

Statement A-11 in addition to four $90^{\circ}$ angles includes their sum $\left(360^{\circ}\right)$. This condition, in addition to being redundant, is not a specific characteristic of a square, but a property of any quadrilateral. In statement A-12, for a "closed figure with four equal sides", the property- "two equal length diagonals" constitutes a sufficient condition for a square; that is, there is no need to refer further to "diagonals crossing each other at a right angle".

By inaccurate terminology we mean using words like 'shape', 'object', or 'figure' when specifying from what larger set of objects squares are drawn. Additional inappropriate terms for the task included "corners", "straight edge", and "diamond". Consider the following examples:

A square is

A-13: A diamond and a rectangle

A-14: A four-sided figure with four equal sides and four equal angles

A-15: A shape with four equal sides where the opposite sides are parallel and the corners meet at right angles

In these three examples, the definition is correct if a 'diamond' is taken to mean a 'rhombus', and a 'four-sided figure' or 'shape' implies a polygon. However, we note that such implications may not necessarily be shared when a more rigorous judgment is applied. Naturally though, more examples of inappropriate terminology were found when the generated definitions were incorrect.

\subsubsection{Inappropriate examples with necessary but not sufficient conditions}

On a number of occasions the request to provide an example of a definition resulted in simply mentioning properties of a square. Though the listed properties were indeed 
Fig. 3 Examples of unintended interpretation of definitions
Objects defined by I-9

Objects defined by I-10

Objects defined by I-11
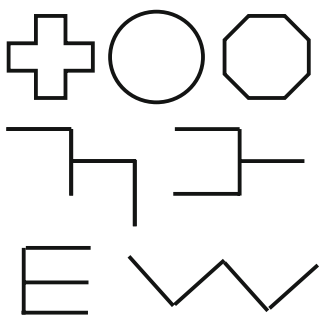

properties of a square, they could not be considered as a definition. Several illustrative examples are listed below:

I-1: There are four sides that have equal length

I-2: Four equal angles, four equal sides

I-3: Opposite sides are parallel to each other

However, in the majority of incorrect examples based on necessary but not sufficient conditions, the object defined was a more general specific quadrilateral-either a parallelogram, a rectangle, or a rhombus, but not a square. Examples included the following:

A square is

I-4: A quadrilateral with equal sides

I-5: A polygon in which the opposite sides are parallel

I-6: A closed geometric shape with four equal sides

I-7: A polygon that has diagonals of equal length

I-8: A polygon with four equal length sides

In other instances a reference to a 'figure' or 'shape', rather than a quadrilateral, allowed for a variety of interpretations of the defined object. We illustrate these unintended interpretations in Fig. 3.

A square is

I-9: A figure that looks the same every time it is rotated $90^{\circ}$

I-10: A shape with four sides that are equal and four $90^{\circ}$ angles

I-11: A figure consisting of four equal sides

\subsubsection{Inappropriate examples with sufficient but not necessary conditions}

Some examples of definitions (five out of 55) generated by participants constituted sufficient but not necessary conditions of a square. These examples described specific cases:

I-12: A square has four vertices placed at $(x, y)(x+1, y)(x, y+1)(x+1, y+1)$

I-13: Two horizontally and two vertically parallel lines of equal lengths forming angles of $90^{\circ}$ at their intersection

Example I-12 unnecessarily restricts the orientation and the size of a square in a (presumably Cartesian) coordinate system. We further note that this formulation is not precise enough for a definition of a square: Though the four points placed as described do 
define four vertices of a square, it is implicit how these points are connected. Example I-13 unnecessarily restricts the orientation. In addition, the usage of the term 'lines' is problematic. Whereas the participant obviously implied 'line segments', 'a line' is conventionally interpreted as 'an infinite straight line'.

\subsubsection{Inappropriate examples with neither necessary nor sufficient conditions}

A number of definitions (seven out of 55) generated by the participants were neither necessary nor sufficient.

I-14: Two parallel horizontal lines perpendicular to two parallel vertical lines.

I-15: A square has four vertices such that exactly two pairs of vertices have the same $x$ coordinates and $y$-coordinates

I-16: Two identical right isosceles triangles joined at the base

I-17: Two congruent isosceles right triangles which share a common hypotenuse

I-18: Two-dimensional cube

Similarly to I-12 and I-13, examples I-14 and I-15 unnecessarily restrict the orientation of the defined object. Additionally, the use of term 'line' without reference to the length of the segments is ambiguous and makes conditions in the sentence insufficient. The coordinates in I-15 specify a rectangle rather than a square. The conditions in examples I-16 and I-17 are neither sufficient nor necessary as the shape of a square does not include a diagonal. I-18 includes internal inconsistency, as a cube is a three-dimensional object.

In summary, considering correctness and accessibility of the collective example space of this group of prospective teachers, we note that of the 40 participants in the study there were only five whose list of examples included only appropriate definitions. However, 26 out of 40 provided at least one appropriate definition. Additionally, we found that providing a greater number of examples did not necessarily mean generating more correct definitions. In fact, the desire to comply with the task by generating more examples often resulted in appropriate definitions provided within the first one or two examples, followed by inappropriate definitions in the examples that followed. Thus, we suggest that the category of accessibility needs reconsideration.

\subsection{Richness}

Since most of the definitions (both correct and incorrect) referred to equal sides and right angles, we consider as 'rich' those definitions that referred to other attributes. Only 35 definitions provided by 21 participants fit in this category. Statements R-1 to R-8 are examples of appropriate definitions that contribute to the richness of an example space, though some require modification of terminology to conform to rigor:

A square is

R-1: A parallelogram with equal and perpendicular diagonals

R-2: Rhombus with equal diagonals

R-3: Rectangle with perpendicular diagonals

R-4: A quadrilateral whose diagonals bisect each other at right angles into four equal size segments

R-5: A rectangle in which area equals to the square of one side

R-6: A rectangle in which the length of one side equals to square root of its area

R-7: A four-sided perimeter containing the maximum area that perimeter can hold 
R-8: The shape that results when four chords of equal length are drawn end-to-end inside a circle

Some rich examples of definitions appeared inappropriate, but "fixable" with minor alteration. The following examples illustrate this category:

A square is

R-9: A quadrilateral whose diagonals are equal and intersect at right angles

R-10: An object inscribed in a circle which has exactly four points touching the circumference and the interior angles of the object all equal $90^{\circ}$

R-11: Can be constructed using a circle with perpendicular diameters, then connecting the four points that touch the circle

R-12: Two line segments are bisectors of each other, connect those four points to form a square

The property in example R-9 may appear in a scalene quadrilateral, however, this definition can be 'fixed' by adding the condition that the diagonals bisect each other at their intersection. Similarly, R-10 can be 'fixed' by changing the 'object' to 'quadrilateral' and adding that the points on the circumference are equally spread. In R-11 the adjustment is required to specify in what way the four points should be connected. In addition to specifying the way of connecting the points, R-12 needs to list another property to define a square rather than a parallelogram.

In contrast, R-13 is an example of a participant's attempt to generate a rich definition beyond remedy. This is because the example brings in terminology from graph theory which is out of scope.

R-13 a graph with four vertices and four edges such that the 'in' and 'out' degree of each vertex is 1 and each edge is perpendicular to every adjacent edge

Further, we believe that the following definitions that explicitly refer to the interior of a square contribute to the richness of the example space.

A square is

R-14: Enclosed area with four equal sides, each side joining at $90^{\circ}$ angle

R-15: One face of a cube

R-16: A slice of a cube having infinitely small thickness

We point out that in order to be a square the 'slice' mentioned in definition R-16 should be parallel to one of the faces; however, this observation belongs to the discussion of correctness and rigor, and not to our current discussion of richness.

We note that conventionally a square is defined as a polygon/quadrilateral with certain properties, which entails that only the boundary, that is the line segments, form the shape. However, this definition is convoluted when used in reference to polyhedra. That is, if a face of a polyhedron is a polygon - and specifically, a face of a cube is a square - then the reference is definitely to the area enclosed in the boundary rather than to the boundary itself. Further, the sources are inconsistent in defining a polygon. It is defined as either "a closed plane figure with $n$ sides" (Weinstein 2007a) or as "a closed curve composed of line segments" (e.g., Musser, Burger and Peterson 2006). While the latter definition (curve) excludes the interior, the former one (figure) can be interpreted either way.

However, this distinction does not usually arise in school when considering Euclidean geometry, and the discussion of properties of polygons is not affected by whether one thinks of its boundary or includes its interior. Further, among the variety of definitions 
mentioned so far, we singled out a few that explicitly include the interior of a square. However, whether or not the interior is considered in a popular definition, such as "a rectangle with equal sides", depends on a concept-image of a rectangle, and in turn of a polygon, which, as we mentioned, is inconsistent.

\subsection{Generality}

Because of the nature of the task, this category has already been attended to in previous analyses. Examples of definitions that do not define a square in general, but in its reference to a particular special orientation, were discussed as inappropriate or incorrect in a previous section. So were the examples that refer to an object more general than a square, such as rectangle or rhombus.

Several statements provided by participants answered the question "what a square is"- "a square is a geometric shape" or "a square is a special case of a rectangle"-rather than formulated a definition. The issue of generality based on these examples, as well as other cases of disagreement in responses to Task 2, generated a conversation on "what is a definition" or "what does it mean to provide a definition for a mathematical concept". We describe the highlights from this conversation in the next section.

\section{Findings: Task 2 or what have we learned?}

As mentioned above, in Task 2 the participants were presented with 24 examples for a definition of a square and asked to consider their validity. The definitions on the list included several "expert" definitions (see Fig. 1) but were mostly taken to illustrate various categories from the examples generated by the group. After individual decisions, the definitions that caused disagreement among the participants were addressed. The exercise generated considerable discussion, and we share below some of the main issues that arose.

\subsection{Usage of 'prior' concepts}

Surprisingly, definitions such as (a) "rectangle with equal sides" or (b) "rhombus with right angles" generated considerable disagreement within the group. While some participants claimed that (a) was THE definition of a square, others referred to is as "cheating", objecting to the usage of "rectangle" or "rhombus", or any prior terms in defining a square. Part of this concern, exemplified by Jody, was based on a desire to avoid circularity:

Jody: If you allow a square to be a rectangle with equal sides, you may then also allow a rectangle to be a square squished, where, if you want, two opposite sides get shorter than the other two, and once you allow for it you will never get out of this relying on one another.

Jody raises an important issue: we cannot define $X$ with reference to $Y$ and at the same time define $Y$ with reference to $X$. However, in this particular case, her claim and her objection to the definition signify her confusion between necessary and sufficient conditions and about the 'subset relationship' between squares and rectangles. A confusion with respect to the use of different 'prior' concepts was also expressed by Rachel:

Rachel: Does a definition that starts with parallelogram or rectangle count? If yes, I have a lot. 
Further, participants who objected to defining a square in terms of a rectangle, agreed to the definitions in terms of a polygon or a quadrilateral. When it was pointed out that these also were 'prior terms', they modified their claim by suggesting that one quadrilateral could not be defined in terms of another quadrilateral. As the classroom deliberation continued, the group of participants who initially claimed these definitions were not 'valid' eventually accepted their mathematical validity and softened their objection. However, they maintained their view that these definitions were not 'preferable' for students, shifting the discussion from mathematical to pedagogical considerations. Since the focus of the exercise was mathematical, rather that pedagogical, the issue of pedagogical preference was not actively pursued. However, despite the instructor's desire to keep the focus on mathematics, attention to pedagogy surfaced yet again with respect to minimality of a definition.

\subsection{Minimal definition versus accessible definition}

The definition: "A square is a rhombus with a right angle" created considerable disagreement. This was expected, as the data generated by this group of participants included 26 barely-non-minimal definitions that referred to "all angles of $90^{\circ}$ ". Moreover, among the 41 definitions classified as "rigorous and minimal", only 13 mentioned "one right angle". Participants who did not accept this definition as valid were readily convinced by a classmate that in the case of a rhombus or a parallelogram 'one right angle' implied 'all right angles', but this realization did little to change their minds with respect to validity of this definition. A pedagogical concern guided their argument. The following conversation, with rather emotional overtone, illustrates the disagreement.

Jack: You want a student to understand the definition and not to struggle with it. When you say "one right angle" they will think one is enough and not even try to think what about other angles.

Jill: But then you say, draw it. Make it with four equal sides and one $90^{\circ}$ angle, and they will see that this works only if all angles get 90 .

Jack: Ok, you do this, if you work with gifted or something. But the students I saw, you do not want to confuse them, you want to give them what they need.

It has been shown in prior research (Leikin and Levav-Waynberg 2007) that the mathematics of practicing teachers is embedded in their teaching practice. However, it is not obvious to find out that prospective teachers' discourse already includes pedagogical considerations and their reasoning is situated in teaching. The above excerpt illustrates that Jack judges validity of definitions not in terms of mathematical criteria, but in terms of accessibility to students, having in mind particular students that he interacted with in his teaching practicum. Pedagogical considerations were also voiced with respect to additional issues, as we describe below.

\subsection{Rigor versus pedagogical consideration}

Several participants' definitions that can be interpreted in ways not intended by the writer were explicitly included in task 2 (see Figs. 2 and 3). However, "a figure with four sides that are equal and four $90^{\circ}$ angles" was initially believed by the majority to be an appropriate definition. The instructor's goal was to alert participants to the necessity of rigor. In this specific example, the figure must be closed, to determine a square, while there 
is no necessity to mention closure when the reference is to a polygon or a quadrilateral, since these shapes are closed "by definition". Further, the 'sides' must be line segments.

Yet again, there were strong voices advocating clarity of a definition and accessibility for students, at the expense of mathematical rigor. It was mentioned that a 'figure' is much clearer for a student than 'closed figure' or 'polygon'. It was also mentioned that an accompanying drawing would in most cases clarify a possible confusion in wording, and therefore, as Janet mentioned, "there is no need to confuse a student with extra words or unfamiliar words".

Further discussion revolved around availability of a drawing in a definition, whether it was a part of a definition or an extension clarifying a definition for students. The mathematical argument - that a drawing cannot be a part of a concept definition as each drawing includes non-essential features of a concept - seemed to be of lesser importance to the participants who advocated for the inclusion of a drawing for pedagogical clarification.

Moreover, two 'expert' definitions in terms of loci (see Fig. 1, items 12 and 13) caused considerable discomfort to participants who advocated clarity and accessibility for students. The instructor's comment, that these statements are equivalent to the familiar definitions, and that they were introduced as an exercise for prospective teachers rather than students, was not attended to. While some participants appreciated the exposure to unconventional definitions, others repeatedly questioned their pedagogical applicability.

\subsection{Is inside included?}

An interesting conversation emerged in class - and continued without resolution among ourselves and our colleagues - with respect to the proposed definition of a square as "one face of a cube". While a face of a cube is definitely a square, is it an appropriate definition? As a working assumption, let us suppose that a cube was defined without referencing a square, so circularity is not of concern. Then the issue is whether a "face of a cube" is a two- or three- dimensional object. Alerted to this possible concern, one participant suggested "infinitely small thickness". However, whether infinitely small is equivalent to zero, which is the 'thickness' of two-dimensional shapes, is a matter of perspective.

Further, as was mentioned in Section 4.2, the concern becomes whether a square includes its interior or is a four-sided boundary only. As mentioned previously, the definitions in different reference sources do not provide an unequivocal resolution. However, having in mind different images of polygons, either including or excluding the interior, does not create a conflict when properties are discussed or theorems are proved. The exercise of exemplifying a definition for a square increased the participants', as well as our own, awareness of a potential discrepancy in interpretation.

\section{Conclusion}

In this study we re-evaluated the claim made in our prior research, that examples generated by participants "mirror their conceptions of mathematical objects involved in an example generation task, their pedagogical repertoire, their difficulties and possible inadequacies in their perceptions" (Zazkis and Leikin 2007, p. 15). We considered to what extent examples generated by participants revealed their knowledge and understanding. The specific contributions of our study can be seen in several arenas: It provides further insight into prospective teachers' ideas and understandings related to the mathematical concept of a square and the meta-mathematical concept of a definition; it shows how the examples 
generated by participants can serve as a lens on their understanding of the concepts; and it examines and provides tools for the refinement of the framework designed for analyzing examples and example spaces.

It is obvious that prospective secondary school teachers, holding degrees in mathematics and science 'know' what a square is. In fact, every kindergartener knows this, in that $\mathrm{s} / \mathrm{he}$ is able to distinguish square-like shapes from others. So what was revealed by participants' examples for definitions of a square? We believe that what was revealed is the ability (or inability) to distinguish between necessary and sufficient conditions, the ability to apply appropriate mathematical terminology and, most importantly, the task revealed the participants' ideas about what a definition is. We believe that simply asking "what is a mathematical definition" could not have generated such an abundant source of data. Nevertheless, exemplifying definitions reveals one's 'answer in action' and illuminates deficiencies in this answer.

With respect to the framework used to classify and analyze participants' examples, we suggest that the categories of correctness and richness provide a clear organizational lens. We also found that 'accessibility', which we initially connected to 'correctness' is a separate rubric that cannot be assessed within a written questionnaire. That is because some easily accessible examples were incorrect and also, with no time limit for the task, we cannot judge to what degree the generated examples were accessible. With respect to 'richness', we found that many attempts to introduce richness resulted in inappropriate definitions. As such, a delicate pedagogical balance should be found between encouraging rich examples while keeping an eye on their appropriateness. We also noted that 'generality' of an example was not a separate measure for the specific task in hand, since the notion of generality is included within a definition itself. While the framework served our purpose, its further refinement and applicability should draw upon a greater variety of tasks.

Having analyzed common deficiencies in the presented examples, we further demonstrated that participant-generated examples serve as a springboard for a valuable pedagogical deliberation. The strategy of asking students to evaluate definitions presented to them in order to investigate their conceptions of a mathematical definition was used in prior research (e.g., Zaslavsky and Shir 2005). Further, it is a common pedagogical strategy to ask students to respond to ideas presented by their classmates. However, the novelty in our study is in combining these two approaches, that is, most of the examples of definitions presented to participants were drawn from the collective example space generated by the group. As such, unlike the case in the Zaslavsky and Shir study, about half of the definitions on our list were invalid mathematically. We believe that this caused each example to be examined with a greater scrutiny.

Examination of classmate-generated examples of definitions revealed strong preference towards pedagogical considerations versus mathematical ones. That is, while the question presented was "which examples from the list are valid definitions of a square?", many participants in their arguments implicitly reformulated the question to "which of the following definitions would I use with my students?". While the didactical considerations in the choice of definitions have been recognized by different researchers (see Section 2), it appears that for many prospective teachers of mathematics in our study pedagogical issues were acknowledged instead of, rather than in addition to, mathematical ones.

Pedagogical considerations of suitability of definitions for a learner (mentioned in Section 2) presuppose that definitions are chosen by the teachers or curriculum designers. However, Freudenthal (1973) strongly criticized this traditional instructional practice, which in his opinion denies students' participation in the mathematical enterprise, and suggested that students should be encouraged to create their own definitions as part of their 
learning. De Villiers (1998) engaged students in constructing their own formal definitions for geometric concepts and demonstrated positive results of this experience. Edwards (1997) recognized the pedagogical benefits of this method in helping students achieve better understanding of mathematics and better appreciation of mathematical activity, but also called for caution referring to this method as 'potentially problematic'. Her research demonstrated that some defining activities for students reinforce the notion that definitions could be 'right' or 'wrong' and that the task of defining is to discover the 'right' definition for a concept.

Our study adds another caution to this approach: In order to be able to guide students through the experience of generating definitions, their teachers should have the ability to engage in this process themselves. It should be noted that our goal is not to advocate a specific definition from either a mathematical or a pedagogical perspective, but to alert the reader to the existing variety in teachers' considerations and choices. As our results demonstrate, prospective teachers' ability to generate examples of definitions varies considerably, even when the task relates to a very familiar concept of a square. If teachers' knowledge is a prerequisite for the success of their students, the tasks described in this research constitute a valuable activity, both mathematical and pedagogical, to promote a deeper conceptual understanding of mathematics in general and of the nature and role of definitions in particular.

\section{References}

Borasi, R. (1992). Learning mathematics through inquiry. Portsmouth, NH: Heinemann Educational Books. Cooney, T. J., \& Wilson, M. R. (1993). Teachers' thinking about functions: Historical and research perspectives. In T. A. Romberg, E. Fennema, \& T. P. Carpenter (Eds.), Integrating research on the graphical representation of functions (pp. 131-158). Hillsdale, NJ: Lawrence Erlbaum.

Dahlberg, R. P., \& Housman, D. L. (1997). Facilitating learning events through example generation. Educational Studies in Mathematics, 33(3), 283-299.

De Villiers, M. (1998). To teach definitions in geometry or to teach to define? In A. Olivier, \& K. Newstead (Eds.), Proceedings of the 22nd Conference of the International Group for the Psychology of Mathematics Education (vol. 2, pp. 248-255). Stellenbosch: RSA.

Edwards, B. (1997). An undergraduate student's understanding and use of mathematical definitions in real analysis. In J. Dossey, J. Swafford, M. Parmantie, \& A. Dossey (Eds.), Proceedings of the 19th Annual Meeting of the North American Chapter of the International Group for the Psychology of Mathematics Education (vol. 1, pp. 17-22). Colulmbus, OH: The ERIC Clearing house for Science, Mathematics, and Environment Education.

Edwards, B. S., \& Ward, M. B. (2004). Surprises from mathematics education research: Student (mis)use of mathematical definitions. The American Mathematical Monthly, 111(5), 411-424.

Fischbein, E. (1987). Intuition in science and mathematics. An educational approach. Dordrecht: Kluwer.

Freudenthal, H. (1973). Mathematics as an educational task. Dordrecht: Reidel.

Khinchin, A. Y. (1968). The teaching of mathematics. London: The English Universities Press.

Leikin, R., \& Levav-Waynberg, A. (2007). Exploring mathematics teacher knowledge to explain the gap between theory-based recommendations and school practice in the use of connecting tasks. Educational Studies in Mathematics, 66(3), 349-371.

Leikin, R., \& Winicky-Landman, G. (2000). On equivalent and nonequivalent definitions II. For the Learning of Mathematics, 20(2), 24-29.

Leikin, R., \& Winicky-Landman, G. (2001). Defining as a vehicle for professional development of secondary school mathematics teachers. Mathematics Teacher Education and Development, 3, 62-73.

Linchevsky, L., Vinner, S., \& Karsenty, R. (1992). To be or not to be minimal? Student teachers' views about definitions in geometry. In W. Geeslin, \& K. Graham (Eds.), Proceedings of the 16th Conference of the International Group for the Mathematics Education (vol. 2, pp. 48-55). Durham, New Hampshire.

Mariotti, M. A., \& Fischbein, E. (1997). Defining in classroom activities. Educational Studies in Mathematics, 34(3), 219-248. 
Mason, J. (1998). Researching from the inside in mathematics education. In A. Sierpinska, \& J. Kilpatrick (Eds.), Mathematics Education as a research domain: A search for identity (pp. 357-377). Dordrecht: Kluwer.

Musser, G. L., Burger, W. F., \& Peterson, B. E. (2006). Mathematics for elementary teachers: A contemporary approach. New York: Wiley.

Pimm, D. (1993). Just a matter of definition. Educational Studies in Mathematics, 25, 261-277.

Poincare, H. (1909/1952). Science and method. New York, NY: Dover.

Solow, D. (1984). Reading, writing and doing mathematical proofs. Book I. Palo Alto, CA: Dale Seymour.

Tall, D., \& Vinner, S. (1981). Concept image and concept definition in mathematics - With particular reference to limits and continuity. Educational Studies in Mathematics, 12, 151-169.

Tirosh, D., \& Even, R. (1997). To define or not to define: The case of $(-8)^{1 / 3}$. Educational Studies in Mathematics, 33(3), 321-330.

van Dormolen, J., \& Zaslavsky, O. (2003). The many facets of a definition: The case of periodicity. Journal of Mathematical Behavior, 22(1), 91-106.

Vasco, C. E. (2006). Cronotopía: Un "Programa de Bogotá" para lo que se suele llamar "Geometría". In C. Ruiz, et al. (Eds.), Memorias: XVI Encuentro de Geometría y sus aplicaciones-IV Encuentro de aritmética (vol. 1, pp. 1-28). Bogotá: Universidad Pedagógica Nacional.

Vinner, S. (1991). The role of definitions in the teaching and learning of mathematics. In D. O. Tall (Ed.), Advanced Mathematical Thinking (pp. 65-81). Dordrecht: Kluwer.

Vinner, S., Linchevski, L., \& Karsenty, R. (1993). How much information should include a geometrical definition? Zentralblatt für Didaktik der Mathematik, 25, 164-170.

Vygotsky, L. S. (1982). Mishlenie i rech (Thought and language). In L. S. Vygotsky (Ed.), Sobranie Sochinenii, t.2. Moscow: Pedagogika (in Russian).

Watson, A., \& Mason, J. (2005). Mathematics as a constructive activity: Learners generating examples. Mahwah, NJ: Lawrence Erlbaum.

Weinstein, E. (2007a). Wolfram MathWorld: Polygon. Retrieved March 15, 2008 from http://www. mathworld.wolfram.com/Polygon.html.

Weinstein, E. (2007b). Wolfram MathWorld: Square. Retrieved March 15, 2008 from http://www.mathworld. wolfram.com/Square.html.

Winicky-Landman, G., \& Leikin, R. (2000). On equivalent and nonequivalent definitions I. For the Learning of Mathematics, 20(1), 17-21.

Zaslavsky, O., \& Shir, K. (2005). Students' conceptions of a mathematical definition. Journal for Research in Mathematics Education, 36(4), 317-346.

Zazkis, R., \& Leikin, R. (2007). Generating examples: From pedagogical tool to a research tool. For the Learning of Mathematics, 27, 11-17. 